\title{
EXCRECIÓN URINARIA DE SODIO EN NIÑOS Y ADULTOS DE UNA COMUNA DE LA REGIÓN METROPOLITANA DE SANTIAGO DE CHILE
}

\section{URINARY SODIUM EXCRETION IN CHILDREN AND ADULTS FROM A BOUROUGH OF THE METROPOLITAN REGION OF CHILE}

\author{
Guadalupe López-Rodríguez (1,2), Marcos Galván-García (1,2), Santiago Muzzo B. (1)
}

(1) Instituto de Nutrición y Tecnología de Alimentos (INTA), Universidad de Chile, Santiago, Chile. (2) Área Académica de Nutrición, Universidad Autónoma del Estado de Hidalgo, México.

\begin{abstract}
Arterial hypertension has increased sharply in Chile, during the last 3 decades. The National Health Survey (2003) registered a $33.7 \%$ prevalence in Chilean population over 17 years of age. It is known that an excessive sodium intake is a risk factor for arterial hypertension (AH). Objective: To measure urinary sodium excretion in an exploratory study in school age children and adults of a borough of the Metropolitan Region, and later on increase to a representative population sample. Subjects and method: 158 school age children of both sexes aged $10.6 \pm 2.5$ years and 48 adults older than 20 years were evaluated. Weight was measured in a $0.1 \mathrm{~kg}$ precision scale (SECA model 286). Body Mass index (BMI) was calculated according to the formula weight (kg)/stature (m)2. Arterial pressure was measured with a mercury manual sphygmomanometer. Urinary sodium in 24 hours was calculated with the formula of Tanaka et al. This information was used to predict theoretical NaCl intake using the equation $\mathrm{Na} \mathrm{mg} / \mathrm{day}=\mathrm{Na} \mathrm{mEq} / \mathrm{d} \times 23 ; \mathrm{NaCl}=\mathrm{Na} \mathrm{g} / \mathrm{d} \times 100 / 39.3$. Results: $\mathrm{BMI}$ in children was $19.2 \pm 4,9$ and $29.0 \pm 5.9$ in adults. In $28.6 \%$ and $40.5 \%$ of adults diastolic and systolic pressure were higher than $90 \mathrm{~mm} \mathrm{Hg}$ and over 130 mm Hg, respectively. In $1.7 \%$ and $0.9 \%$ of children systolic and diastolic pressure were high, according to age and sex. Salt intake per day was calculated assuming that all sodium ingested was consumed as $\mathrm{NaCl}$. Children $\mathrm{NaCl}$ intake was $3 \pm 2.2 \mathrm{~g}$ per day and in adults was $10.4 \pm 2.5 \mathrm{~g}$ per day. Conclusions: BMI was elevated in children and adults in accordance with the high obesity prevalence in the country. The high percentages of elevated blood pressure present in adults were in accordance with high prevalence of hypertension in Chilean adults. Salt intake was high in children and adults constituting a risk factor of hypertension in our population.

Key words: arterial hypertension, salt intake, obesity, children, adults.
\end{abstract}

Este trabajo fue recibido el 26 de Junio de 2009 y aceptado para ser publicado el 10 de Noviembre de 2009.

El sodio es un electrolito esencial para la nutrición humana, y la sal $(\mathrm{NaCl})$ es la mejor fuente en la dieta. $\mathrm{El} \mathrm{NaCl}$ se ha utilizado durante siglos para realzar el sabor y conservar alimentos, pero su ingesta excesiva se considera un factor de riesgo para el desarrollo de hipertensión arterial (HTA) y enfermedades coronarias $(1,2)$. Recomendaciones internacionales y nacionales del Ministerio de Salud (MINSAL) indican restringir su consumo como medida preventiva de la HTA, para el manejo de pacientes hipertensos y/o con enfermedades renales (3).

En la nutrición humana el consumo de sal representa una paradoja, debido a que se requiere ingerirla para evitar el déficit de sodio y las enfermedades asociadas a este nutriente; además que la sal es utilizada en muchos países como vehiculo para la suplementación de otros nutrientes que representan un problema de salud publica, como es el caso del yodo (4); sin embargo, la ingesta excesiva de sal se asocia al desarrollo de hipertensión y otras enfermedades crónicas (5), y la población ha asimilado el mensaje de que es un alimento dañino para su salud.

Debido al efecto negativo del sodio en la presión arterial, el MINSAL de Chile ha discutido la importancia de una ingesta adecuada de sal como un factor que puede estar asociado al cambio en el perfil epidemiológico de 
la población (6). En los últimos 30 años en Chile, la proporción de sujetos con hipertensión se incrementó de forma muy importante, en el año 2003 la Encuesta Nacional de Salud registró que esta enfermedad afectaba al $33.7 \%$ de la población Chilena $\geq 17$ años, la frecuencia de HTA era menor en jóvenes de 17-24 años (5.6\%) y mayor en los adultos > 65 años (78.8\%) (7).

Para tratar de conocer si existe una asociación entre la ingesta de sodio y el incremento de la HTA en la población Chilena, es de interés realizar primero un estudio exploratorio y descriptivo donde se determine la ingesta de sal y excreción de sodio en orina en un grupo de la población, para que en caso de encontrar una alta ingesta, proseguir con una muestra representativa poblacional. Por lo tanto, el objetivo de este trabajo fue evaluar estas variables en escolares de educación básica de la comuna del Bosque de la Región Metropolitana de Santiago y en adultos sanos de dicha comuna.

\section{SUJETOS Y MÉTODO}

Se realizó un estudio transversal, descriptivo y exploratorio en escolares de educación básica y sus padres, de la comuna del Bosque de la Región Metropolitana de Santiago. La población de estudio se eligió aleatoriamente dentro de los colegios por cada nivel educacional. Los alumnos seleccionados y sus padres ( $\geq 20$ años) fueron invitados a participar en el estudio a través de una comunicación enviada a sus domicilios. Los criterios de exclusión fueron: a) cursar con enfermedad crónica diagnosticada previamente por su médico tratante; b) presentar algún proceso infeccioso al momento del estudio.

El tamaño de la muestra de adultos se calculó asumiendo que un tercio de ellos presentan hipertensión y consumen alrededor de $10 \mathrm{~g}$ de sal por día, con un error alfa de 0.01 , una desviación estandar de $15 \%$ y un poder de $90 \%$. El N calculado fue de 47.

Antes de tomar la muestra se solicitó la firma de un consentimiento informado a los apoderados de los escolares y a los adultos participantes en este estudio.

Se evaluaron 158 niños en edad escolar y 48 adultos mayores de 20 años. Todos los participantes en el estudio se pesaron y midieron utilizando técnicas estandarizadas (8). El peso se determinó usando una báscula con precisión de $0.10 \mathrm{Kg}$ (SECA, modelo 286) y la talla fue tomada con el sujeto de pie utilizando un estadiómetro portátil con precisión de $1 \mathrm{~mm}$ (SECA, modelo 208). El Indice de Masa Corporal (IMC) se calculó con la ecuación: peso $(\mathrm{Kg}) /$ talla2. La presión arterial se midió con un esfigmomanómetro manual de mercurio, con el paciente sentado, colocando el brazo del paciente en una superficie fija, la fosa cubital al nivel del corazón, con adaptaciones sugeridas en los niños (9). La excreción urinaria de sodio se determinó en una muestra casual de orina (10) colectada entre las 8:00 y las 10:00 horas. Los valores de sodio corregidos se reportan en relación a la creatinina urinaria (11).

La excreción urinaria de $\mathrm{Na}$ en 24 horas fue calculada utilizando la ecuación propuesta en el trabajo de Tanka T y col (12). Este dato se utilizó para predecir la ingesta teórica de $\mathrm{NaCl}$, utilizando las siguientes ecuaciones: $\mathrm{Na} \mathrm{mg} / \mathrm{d} i ́ \mathrm{a}=\mathrm{Na} \mathrm{mEq} / \mathrm{d}$ x 23, $\mathrm{NaCl}=\mathrm{Na}$ g/d $\mathrm{x}$ 100/ 39.3.

Las diferencias estadísticas entre niños y niñas se determinarán por un $t$ de Student.

\section{RESULTADOS}

Se evaluaron 158 escolares cuya edad promedio fue de 10.6 \pm 2.5 años, de los cuales 72 correspondían al sexo femenino y 86 al masculino; los adultos analizados fueron 48 (43 mujeres y 5 hombres). Las características generales de los participantes de estudio se describen en la tabla 1 en la cual se aprecia los altos promedios de IMC en niños y adultos que concuerdan con las altas tasas de sobrepeso y obesidad presentes en nuestra población. En el $28.6 \%$ y en el $40.5 \%$ de los sujetos adultos evaluados

\section{TABLA 1}

\section{Variables descriptivas de la población evaluada}

\begin{tabular}{lcc} 
& Niños & Adultos \\
\hline N & 158 & 48 \\
Edad (años) & $10.6 \pm 2.5$ & $39 \pm 7$ \\
IMC & $19.2 \pm 4.9$ & $29.0 \pm 5.9$ \\
Presión sistólica (mm Hg) & $103.9 \pm 11.9$ & $121.5 \pm 13.1$ \\
Presión diastólica (mm Hg) & $66.4 \pm 9.4$ & $79.5 \pm 11.8$
\end{tabular}


se registró una cifra alta de de presión diastólica $(\geq 90$ $\mathrm{mm} \mathrm{Hg}$ ) y sistólica $(130 \geq \mathrm{mm} \mathrm{Hg})$, respectivamente. En los niños la frecuencia de presión elevada para la edad y sexo, de acuerdo a la presión sistólica (1.7\%) y la diastólica $(0.9 \%)$ fue muy baja.

Se observó que los valores de la excreción de $\mathrm{Na} / \mathrm{L}$ y el Na:Creatinina $(\mathrm{g} / \mathrm{g})$ proveniente de una muestra casual de orina son muy semejantes en los niños, con una pequeña variación en adultos. No se observaron diferencias significativas para las variables evaluadas entre niños y niñas, excepto en la excreción de $\mathrm{Na}$ :Creatinina $\mathrm{g} / \mathrm{g}$, valor que fue mayor en la niñas, $3.02 \pm 1.8$ y $3.8 \pm$ 2.4 respectivamente.

En relación con la cantidad de $\mathrm{Na} / \mathrm{L}$, todos los niños evaluados excretaron $15 \%$ menos sodio en relación a los adultos, este valor concuerdan con los valores de presión diastólica y sistólica observados en niños, los cuales fueron también $15 \%$ menores en este grupo de edad cuando se compararon con los datos registrados en los adultos. Las diferencias en la excreción de sodio en los niños y adultos incrementan cuando se corrigen los valores de sodio por gramo de creatinina, siendo $21.9 \%$ mayor en los sujetos adultos evaluados (tabla 2).

Partiendo del dato predictivo de la excreción de sodio en 24 horas calculado con la ecuación de Tanka T y col, se calculó la ingesta de sal por día, bajo el supuesto que todo el sodio ingerido por los sujetos de estudio se consumió como $\mathrm{NaCl}$. Los niños evaluados ingirieron $7.3 \pm 2.2$ gramos de $\mathrm{NaCl}$ por día y los adultos $10.4 \pm$ 2.5 gramos de $\mathrm{NaCl}$ por día (tabla 3 ).

\section{DISCUSIÓN}

La excreción de cantidades cercanas a los $150 \mathrm{mEq}$ $\mathrm{Na} /$ día es un hallazgo común en muchos países del mundo (13), en donde se ha incrementado la ingesta de sal y alimentos procesados, este hecho cobra relevancia debido a que desde la década de los 60's se ha informado de una asociación lineal entre el promedio de la ingesta de sal y la presión sanguínea en animales (14) y humanos (15). Por lo tanto es ya una practica común en los países realizar un registro de la ingesta de sal en su población.

El consumo de sodio registrado en los participantes de este estudio supera la ingesta recomendada para estos grupos de edad, considerando que las DRI para sodio son de 1.5 gramos por día en los niños y adultos (16), y el nivel máximo de ingesta que no implica riesgo para la salud esta marcado entre 2.2-2.3 g/día. La American Health Association recomienda que el consumo diaria de sal para adultos debe ser de $6.0 \mathrm{~g}$ /día (2.4 gramos Na/ día) con el fin de prevenir la hipertensión, de acuerdo a esta recomendación, los adultos evaluados en este

\section{TABLA 2}

\section{Excreción urinaria de sodio a partir de una muestra casual de orina}

\begin{tabular}{lcc}
\hline & Niños & Adultos \\
\hline $\mathrm{Na} \mathrm{g} / \mathrm{L}$ & $3.4 \pm 1.5$ & $4.0 \pm 1.4$ \\
$\mathrm{Na}: \mathrm{Cr}$ g/g* & $3.4 \pm 2.1$ & $4.3 \pm 2.5$ \\
$24 \mathrm{HUNaV}(\mathrm{mEq} / \mathrm{d}) \dagger$ & $124.1 \pm 38.6$ & $177.5 \pm 43.4$ \\
\hline * Cr= Creatinina urinaria. & & \\
$\dagger$ Excreción urinaria de sodio/día, calculada con la ecuación $24 \mathrm{HUNaV}=21.98 \times((\mathrm{SUNa} / \mathrm{SUCr} \times 10) \times \mathrm{PRCr})^{0.392}$
\end{tabular}

\section{TABLA 3}

\section{Predicción de la ingesta diaria de $\mathrm{Na}$ y $\mathrm{NaCl}$}

$\begin{array}{lcc} & \text { Niños* } & \text { Adultos* } \\ \text { Ingesta } \mathrm{Na} \mathrm{g/d} & 2.9 \pm 0.9 & 4.1 \pm 1.0 \\ \text { Ingesta } \mathrm{NaCl} \text { g/d } & 7.3 \pm 2.2 & 10.4 \pm 2.5\end{array}$

* La ingesta de sodio fue calculada a partir de los datos de la excreción de Na provenientes de una muestar casual de orina. 
estudio están consumiendo en promedio el $73 \%$ más sodio del recomendado.

El sodio en los alimentos existe en diversas formas, los alimentos procesados contienen además de $\mathrm{NaCl}$ otro tipo de compuesto con sodio. Ejemplos de estos son el benzoato de sodio, citrato de sodio, glutamato monosódico entre otros. Por esta razón, es necesario aclarar que la ingesta calculada de sal en este estudio se realizó considerando que todo el sodio consumido fue en forma de $\mathrm{NaCl}$, sin embargo, el sodio excretado en los sujetos evaluados pudo provenir también de otros compuestos de sodio, como los antes referidos. Se ha calculado que del $50-85 \%$ del sodio cuantificado en la orina de un sujeto proviene de otros compuestos de $\mathrm{Na}$, principalmente contenidos en alimentos industrializados (17-19)

En este estudio no podemos indicar cual es la fuente de sodio ingerida, sin embargo, los datos obtenidos señalan que los niños y adultos están consumiendo cantidades de sodio consideradas de riesgo, esto cobra relevancia debido a que en este estudio el calculó de la ingesta teórica de $\mathrm{Na} / \mathrm{d}$ no consideró el $\mathrm{Na}$ que se pierde en las heces fecales o en el sudor, lo que podría incrementar hasta en $25 \%$ la cantidad sodio ingerido (20). El alto consumo de sodio identificado en la población estudiada pudiera estar explicando la alta incidencia de hipertensión observada en la población Chilena, la que incide en la alta mortalidad por coronariopatías en el país. Consideramos de interés poder continuar estos estudios en una muestra representativa de la población chilena.

\section{RESUMEN}

En las últimas 3 décadas en Chile, la hipertensión arterial se incrementó notoriamente. La Encuesta Nacional de Salud (año 2003) registró que esta enfermedad afectaba al $33.7 \%$ de la población chilena mayor de 17 años. Se conoce que la ingesta excesiva de Na constituye un factor de riesgo para el desarrollo de hipertensión arterial (HTA). Objetivo: Medir la excreción urinaria de $\mathrm{Na}$ en escolares y adultos de una comuna de la Región Metropolitana en estudio exploratorio, para posteriormente ampliarlo a una muestra representativa poblacional. Sujetos y método: Se evaluaron 158 escolares de ambos sexos con edad promedio de $10.6 \pm 2.5$ años y 48 adultos mayores de 20 años. El peso se determinó con una báscula con precisión de $0.10 \mathrm{~kg}$ (SECA, modelo 286). El Indice de Masa Corporal (IMC) se calculó con la ecuación: peso $(\mathrm{kg}) /$ talla $^{2}$. La presión arterial se midió con un esfigmomanómetro manual de mercurio. La excreción urinaria de $\mathrm{Na}$ en 24 horas fue calculada utilizando la ecuación de Tanka T y col. Este dato se utilizó para predecir la ingesta teórica de $\mathrm{NaCl}$, utilizando las siguientes ecuaciones: $\mathrm{Na} \mathrm{mg} / \mathrm{día}=\mathrm{Na} \mathrm{mEq} / \mathrm{d} \times 23$,
$\mathrm{NaCl}=\mathrm{Na}$ g/d x 100/39.3. Resultados: El IMC en niños fue de $19.2 \pm 4.9$ y en adultos de $29.0 \pm 5.9$. En el $28.6 \%$ y en el $40.5 \%$ de los sujetos adultos evaluados se registró una cifra alta de de presión diastólica ( $\geq 90 \mathrm{~mm} \mathrm{Hg}$ ) y sistólica $(130 \geq \mathrm{mm} \mathrm{Hg})$, respectivamente. En $1.7 \%$ de los niños se detectó presión sistólica elevada y en $0.9 \%$ de la diastólica, de acuerdo a la edad y sexo. Se calculó la ingesta de sal por día, suponiendo que todo el sodio ingerido se consumió como $\mathrm{NaCl}$. Los niños evaluados ingirieron $7.3 \pm 2.2$ gramos de $\mathrm{NaCl}$ por día y los adultos $10.4 \pm 2.5$ gramos de $\mathrm{NaCl}$ por día. Conclusiones: $\mathrm{El}$ IMC era elevado en adultos y niños, de acuerdo con las altas tasas de obesidad de nuestra población. Las porcentajes de presión elevada fueron mucho mayores en los adultos y de acuerdo a la alta prevalencia de hipertensión en adultos chilenos. La ingesta de sal fue alta tanto en adultos y niños, constituyendo un factor de riesgo de hipertensión arterial.

Palabras clave: hipertensión arterial, ingesta de sal, obesidad, niños, adultos.

Dirigir la correspondencia a:

Dr.

Santiago Muzzo B.

INTA, Universidad e Chile

Macul 5540

Macul, Santiago

Chile

Fono: 9781500

Fax: 2214030

Correo electrónico: smuzzo@inta.cl

\section{BIBLIOGRAFÍA}

1. Alderman $\mathrm{MH}$. Evidence relating dietary sodium to cardiovascular disease. J Am Coll Nutr. 2006; 25(S3):S256-S261

2. Titze J, Ritz E. J Salt and its effect on blood pressure and target organ damage: New pieces in an old puzzle. Nephrol. 2009 Mar-Apr;22(2):177-89

3. Fishberg AM. Hypertension and Nephritis. Philadelphia: Lea and Febiger; 1939.

4. Burgi H, Zimmermann MB. Salt as a carrier of iodine in iodine deficient areas. Schweiz Monatsschr Zahnmed. 2005;115:648-650

5. Lozada M, Sánchez-Castillo CP, Cabrera GA, Mata II, Pichardo-Ontiveros E, James WP. Salt: its goodness and perversities. Rev Invest Clin. 2007 Sep-Oct;59(5):382-93

6. Albala C, Vio F, Kain J, Uauy R. Nutrition transition in Chile: determinants and consequences. Public Health Nutr 2002;5:123-128.

7. Ministerio de Salud de Chile. Encuesta Nacional de 
Salud 2003 (ENS). Ministerio de Salud, Santiago de Chile 2004.

8. Lohman T, Roche A, Martorell R. Anthropometric standarization reference manual. Champaing, IL: Human Kinetics Books, 1991.

9. Grupo de Hipertensión. Consenso sobre factores de riesgo de enfermedad cardiovascular en pediatría. Hipertensión arterial en el niño y el adolescente. Arch argent pediatr 2005; 103:348-366.

10. Bradbury JT. Simplified method for estimation of sodium. J Lab and Clin Med, 1946;31:1257

11. Biggs HG, Cooper JM. An evaluation of four methods of measuring urinary creatinine. Clin Chem 1961;7:665-73.

12. Tanaka T, Okamura T, Miura K, Kandowaski T, Ueshima $\mathrm{H}$, Nakagawa $\mathrm{H}$ and et al. A simple method to estimate populational 24-h urinary sodium and potassium excretion using a casual urine specimen. J Hum Hypertens 2002;16:97-103.

13. Intersalt Cooperative Research Group. Intersal: an international study of electrolyte excretion and blood pressure. Result for 24 hour urinary sodium and potassium excretion. B Med J 1988; 297:319328

14. Meneely GR. The experimental epidemiology of sodium chloride toxicity in the rat. En: Stamler J, Stamler R, Pullman TN, eds. The epidemiology of hypertension. New York: Grune and Stratton. 1967:240-246

15. Dahl L. Possible role of salt intake in the development of hypertension. En: Cottier P. Bock KD, eds. Essential hypertension: an international symposium. Berlin: Springer-Verlag, 1960:53-65.

16. Dietary Reference Intakes for water, potassium, sodium, chloride, and sulfate. The National Academies Press 2004.

17. Bisi M MC, Cunha R de Sá; Herkenhoff LF. Hipertensão arterial e consumo de sal em população urbana. Rev Saúde Pública 2003;37:743-750.

18. Sanchez-Castello CP, Warrender 5, Whitehead TP, James WPT. An assessment of the sources of dietary salt in a British population. Clin Sci 1987;72:95-102)

19. Mattes RD. Donnelly D. Relative contributions of dietary sodium sources. J Am Coll Nutr 1991: 10: 383-393.

20. Allsopp AJ, Sutherland R, Wood P, Wootton SA. The effect of sodium balance on sweat sodium secretion and plasma aldosterone concentration. Eur J Appl Physiol Occup Physiol 1998;78(6):516-521. 